\title{
Phase Transitions in Communication Networks with Limited Elements of Valence
}

\author{
I. E. Suleimenov, G. A. Mun, S. V. Panchenko, Z. Tasbulatova, and A. Nurtazin
}

\begin{abstract}
It is shown that the communication networks with low-valence elements are able to undergo phase transitions. In the result of them the number of elements having the maximum number of bonds increases discontinuously. Such a phase transition can also be interpreted as a discontinuous transition from set of isolated elements to a branched network.
\end{abstract}

Index Terms - Complex networks, innovation environment, network education, peer education.

\section{INTRODUCTION}

The results, summarized in recent papers [1]-[3], allow to assert at present time that the studying of the evolution of bonds distribution between the elements of the complex system without specification of its nature, acquires scientific significance. In the cited studies have shown that the models constructed on the basis of consideration of the graphs formed by elements with a variable number of bonds between them, are used to solve problems in physics, physical chemistry, biology, ecology and social sciences.

In the cited studies, as well as [4], [5] have shown that the assumption of an increase in the number of bonds per single element leads to effects which are treated as a phase transition that allows us to interpret the qualitative transformation of complex systems, i.e. to reveal the mechanisms of evolution. Simplifying somewhat, we can say that any system, the number of bonds between the elements of which may change over time, for example, under the influence of external factors, it is able to move abruptly from one state to another.

In research works on studying of complex systems, in particular [6], [7], as a rule, are considered elements capable of forming unlimited number of connections. This approach largely emerged for historical reasons, as this type of the system is successfully applied to the quantitative description of interpersonal communication in the Internet [8], the spread of epidemics [9], etc.

At the same time, we are interested in the phase transitions in systems which valence of elements remains limited. An example of this is the formation of hydrophilic interpolymer associates (HIA, [10], [11]). This little-studied class of interpolymer reaction product is a network which exists in

Manuscript received November 25, 2016; revised March 21, 2017.

I. E. Suleimenov, S. V. Panchenko and Z. Tasbulatova are with the Almaty University of Power Engineering and Telecommunications, Baitursynova 126, 050013 Almaty, Kazakhstan.

G. A. Mun is with the Kazakh National University, Al-Farabi 71, 050040 Almaty, Kazakhstan.

A. Nurtazin is with the Narxoz University, Zhandosov 55, 050035 Almaty, Kazakhstan (e-mail: anuar_nurtazin@mail.ru). the dynamic mode, the bonds between the elements of which they are destroyed and formed again, being in dynamic equilibrium. Presumably, products as the HIA are essential for the implementation of the evolutionary mechanisms that preceded the biological evolution.

In this paper, it is shown that phase transitions can occur in the systems that can be represented by the graphs, the vertices of which form a small number of bonds.

\section{MODEL}

The used model is a modification proposed in [4], [5]. It is assumed that each element of the system can form $L$ bonds with other elements, i.e. $\mathrm{L}$ is a valence of the element. The probability of bond formation is proportionally to the number of free valences. It is assumed that the break of the bond can occur spontaneously, which is characterized by frequency of break.

We will use the L-level model, which considers the formation of new bonds between nodes as the transition of a lower level to a higher level (Fig. 1) for the analytical description of such processes.

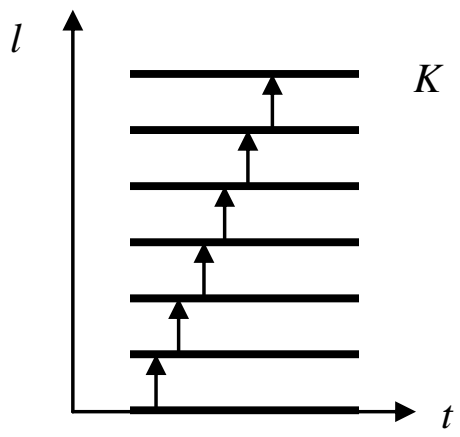

Fig. 1. L-level scheme of transitions.

Formally, this process can be represented by equation similar to the chemical reaction:

$$
[i]+[j] \rightarrow[i+1]+[j+1]
$$

where $[i]$ is the designation of a node with $\mathrm{i}$ bonds (node having valence equal to i)

It is proposed that the capacity of the node to form new bonds depends on the number of bonds already formed. In particular, there may be a limit for the number of bonds for this system - the valence of the separate node.

In the simplest (for analytical description) case, this assumption leads to the following expression for the rate of the process (1)

$$
w_{i j}=w_{0} \alpha^{i} \alpha^{j} N_{i} N_{j}
$$


where the coefficients $\alpha^{i}$ characterize the ability of an individual node to come into the further cooperation, $N^{i}$ is the number of $i$-elements (elements having $i$ bonds), $w_{0}$ is the common denominate multiplier (the characteristic frequency of bonds formation), $w_{i j}$ is frequency of reaction (1). Due to the nature of equation (2) we can consider that $\alpha^{0}=1$.

Equation (2) means that the bond may occur between two nodes capable of forming them in accordance with (1).

Then, increase the number of bonds between nodes can be characterized by equations, describing the transitions between levels in the L-level system. We have

$$
\begin{gathered}
\frac{d N_{0}}{d t}=-w_{0} N_{0} \sum_{j=0} \alpha^{j} N_{j}+v_{1} N_{1} \\
\frac{d N_{k}}{d t}=-w_{0} \sum_{j=0} \alpha^{j} \alpha^{k} N_{j} N_{k}+ \\
w_{0} \sum_{j=0} \alpha^{j} \alpha^{k-1} N_{j} N_{k-1}-v_{k} N_{k}+v_{k+1} N_{k+1} \\
\frac{d N_{K-1}}{d t}=w_{0} \sum_{j=0} \alpha^{j} \alpha^{K-1} N_{j} N_{K-1}-v_{K-1} N_{K-1}
\end{gathered}
$$

where $v_{k}$ is the frequency of spontaneous breaking bond.

In the equations (4) and (5) it is possible to pick out a common multiplier, converting them to a form

$$
\begin{gathered}
\frac{d N_{0}}{d t}=-N_{0} w_{0}\left(\sum_{j=0} \alpha^{j} N_{j}\right)+v_{1} N_{1} \\
\frac{d N_{k}}{d t}=-w_{0}\left(\sum_{j=0} \alpha^{j} N_{j}\right)\left(\alpha^{k} N_{k}-\alpha^{k-1} N_{k-1}\right) \\
-v_{k} N_{k}+v_{k+1} N_{k+1}
\end{gathered}
$$

By the direct summing equations (6) and (7) we can easily show that it must be fulfilled the condition of keeping the total number of elements in the system

$$
\sum_{0}^{\prime} N_{k}=C
$$

At equilibrium case, these equations are obviously acquire the form

$$
\begin{aligned}
& -N_{0} w_{0} \alpha_{0}\left(\sum_{j=0} \alpha^{j} N_{j}\right)+v_{1} N_{1}=0 \\
& -w_{0}\left(\sum_{j=0} \alpha^{j} N_{j}\right)\left(\alpha^{k} N_{k}-\alpha^{k-1} N_{k-1}\right) \\
& -v_{k} N_{k}+v_{k+1} N_{k+1}=0
\end{aligned}
$$

Let

$$
G=\frac{w_{0}}{v_{1}}\left(\sum_{j=0} \alpha^{j} N_{j}\right)
$$

Then these equations can be rewritten by the control parameter as a

$$
\begin{gathered}
N_{1}=G N_{0} \\
-G\left(\alpha^{k} N_{k}-\alpha^{k-1} N_{k-1}\right)-\lambda_{k} N_{k} \\
+\lambda_{k+1} N_{k+1}=0
\end{gathered}
$$

where we use the following substitution

$$
\lambda_{k}=\frac{v_{k}}{v_{1}}, k=2,3, \ldots K
$$

Obviously, in this case, the occupancy of each level in the system is described by following recurrent ratios

$$
N_{k+1}=\frac{1}{\lambda_{k+1}}\left(G\left(\alpha^{k} N_{k}-\alpha^{k-1} N_{k-1}\right)+\lambda_{k} N_{k}\right)
$$

In particular, for the second level we can (4) expression at once

$$
N_{2}=\frac{1}{\lambda_{2}} G\left(\alpha^{1} N_{1}-N_{0}\right)+N_{1}
$$

or

$$
N_{2}=\left(G \frac{\alpha^{1}}{\lambda_{2}}+1\right) N_{1}-G N_{0}
$$

from whence

$$
N_{2}=\frac{\alpha^{1}}{\lambda_{2}} G^{2} N_{0}
$$

The same way,

$$
N_{3}=\frac{1}{\lambda_{3}}\left(\begin{array}{l}
G\left(\alpha^{2} \frac{\alpha^{1}}{\lambda_{2}} G^{2} N_{0}-\alpha^{1} G N_{0}\right) \\
+\lambda_{2} \frac{\alpha^{1}}{\lambda_{2}} G^{2} N_{0}
\end{array}\right)
$$

or

$$
N_{3}=\frac{\alpha^{1} \alpha^{2}}{\lambda_{2} \lambda_{3}} G^{3} N_{0}
$$

\section{Phase Transitions in a Four-LeVEl System}

Let us consider the case of a four-level system, showing that even with the minimum number of levels in the systems, forming bonds, where may be phase transitions. We utilize the expression (8), we have 


$$
C=\frac{\alpha^{1} \alpha^{2}}{\lambda_{2} \lambda_{3}} G^{3} N_{0}+\frac{\alpha^{1}}{\lambda_{2}} G^{2} N_{0}+G N_{0}+N_{0}
$$

From the received ratio and the expression (11) we can obtain an equation on the parameter $\mathrm{G}$, describing the behavior of the system. We have

$$
G=\frac{w_{0}}{v_{1}}\left(N_{0}+\alpha^{1} G N_{0}+\alpha^{2} \frac{\alpha^{1}}{\lambda_{2}} G^{2} N_{0}\right)
$$

Combining (19) and (20) we obtain

$$
\begin{aligned}
& G\left(\frac{\alpha^{1} \alpha^{2}}{\lambda_{2} \lambda_{3}} G^{3}+\frac{\alpha^{1}}{\lambda_{2}} G^{2}+G+1\right)= \\
& C \frac{w_{0}}{v_{1}}\left(1+\alpha^{1} G+\alpha^{2} \frac{\alpha^{1}}{\lambda_{2}} G^{2}\right)
\end{aligned}
$$

At the same time the occupancy of all four levels of the system expressed by parameter $\mathrm{G}$

$$
\begin{aligned}
& \frac{N_{0}}{C}=\frac{1}{\frac{\alpha^{1} \alpha^{2}}{\lambda_{2} \lambda_{3}} G^{3}+\frac{\alpha^{1}}{\lambda_{2}} G^{2}+G+1} \\
& \frac{N_{1}}{C}=\frac{G}{\frac{\alpha^{1} \alpha^{2}}{\lambda_{2} \lambda_{3}} G^{3}+\frac{\alpha^{1}}{\lambda_{2}} G^{2}+G+1} \\
& \frac{N_{2}}{C}=\frac{\frac{\alpha^{1}}{\lambda_{2}} G^{2}}{\frac{\alpha^{1} \alpha^{2}}{\lambda_{2} \lambda_{3}} G^{3}+\frac{\alpha^{1}}{\lambda_{2}} G^{2}+G+1} \\
& \frac{N_{3}}{C}=\frac{\alpha^{1} \alpha^{2}}{\lambda_{2} \lambda_{3}} G^{3} \\
& \frac{\alpha^{1} \alpha^{2}}{\lambda_{2} \lambda_{3}} G^{3}+\frac{\alpha^{1}}{\lambda_{2}} G^{2}+G+1
\end{aligned}
$$

It completes the analytical description of the system, which essentially reduced to the solution of the fourth-order algebraic equation (23).

\section{The Calculation Results}

The calculation results are presented in Fig. 2 - Fig. 8. Fig. 2 shows the dependence of the equation (23) solution from the control parameter

$$
q=C \frac{w_{0}}{v_{1}}
$$

for various values of the other parameters which determine the speed of the transitions between the levels (the frequency of formation and breaking of the bonds).

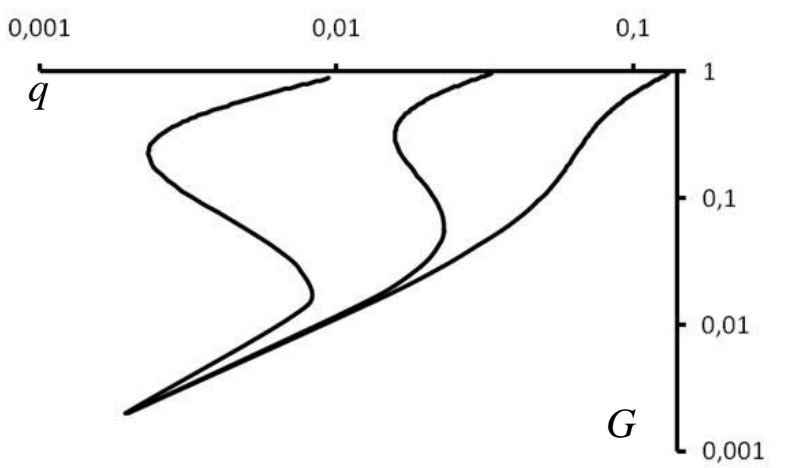

Fig. 2. Dependences of equation (23) solution, the parameter $\mathrm{G}$ of the control parameter q for different values of frequency $\alpha^{1}=10(1), 100(2), 1000$ (3);

$$
\alpha^{2}=10, \lambda_{2}=3, \lambda_{3}=50
$$

We see that at relatively low values of frequency $\alpha^{1}$, which determines the rate of occupancy in the upper level of the system, where obtained solutions are monotone. With the growth of the frequency, curves lose monotony and become S-shaped. That, in particular, suggests the possibility of hysteresis effects occurrence and associated with discontinuous phase transitions.

Such transitions are illustrated Fig. 3-Fig. 8.

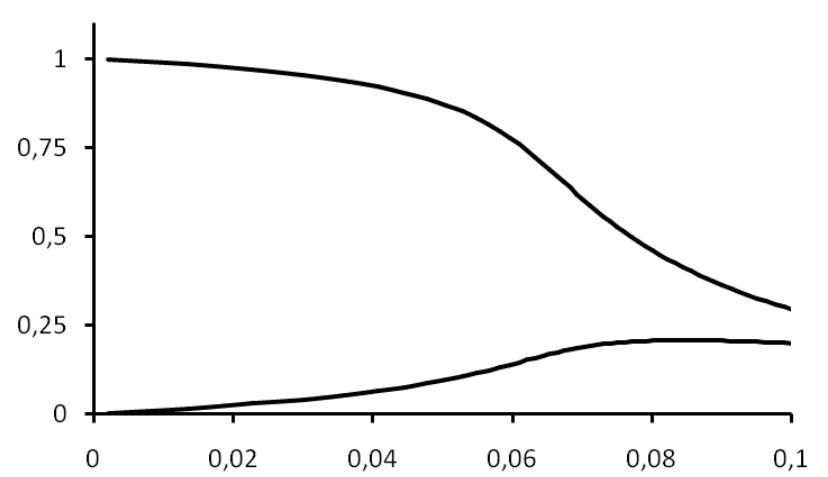

Fig. 3. Dependencies of occupancy of levels 0 (curve 1) and 1 (curve 2) with the control parameter q; $\alpha^{1}=10 ; \alpha^{2}=10, \lambda_{2}=3, \lambda_{3}=50$.

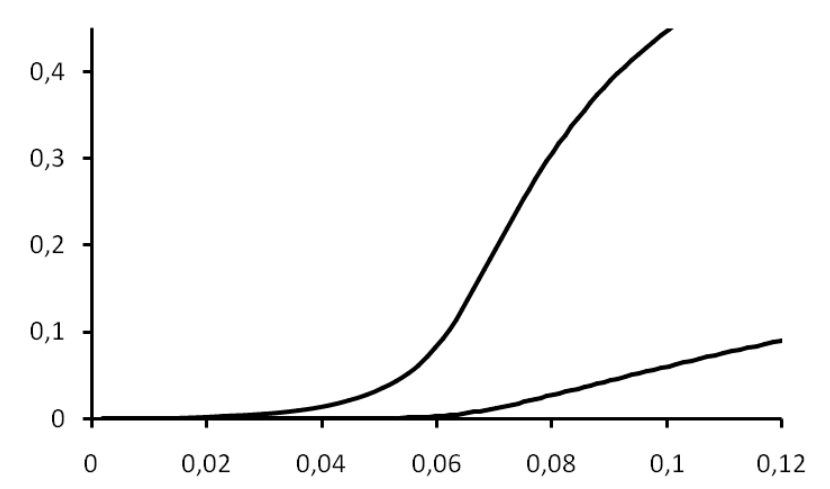

Fig. 4. Dependencies of occupancy of levels 2 (curve 1) and 3 (curve 2) with the control parameter q; $\alpha^{1}=10 ; \alpha^{2}=10, \lambda_{2}=3, \lambda_{3}=50$.

From Fig. 3 and Fig. 4 it is shown that at relatively low 
values of frequency $\alpha^{1}$ depending on all levels occupancy in the system from the control parameter (which can be interpreted as a given number of system elements) is monotonic. In contrast (Fig. 5 - Fig. 8) high values of frequency all considered dependences become S-shaped.

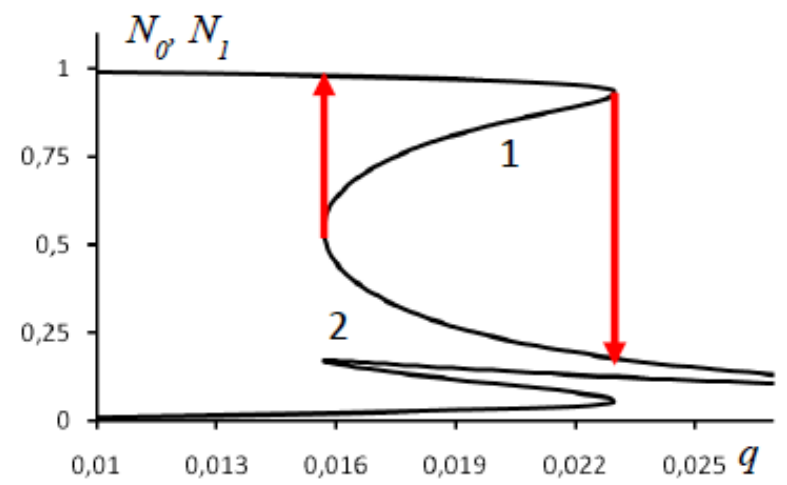

Fig. 5. Dependencies of occupancy of levels 0 (curve 1) and 1 (curve 2) with the control parameter q; $\alpha^{1}=10 ; \alpha^{2}=100, \lambda_{2}=3, \lambda_{3}=50$.

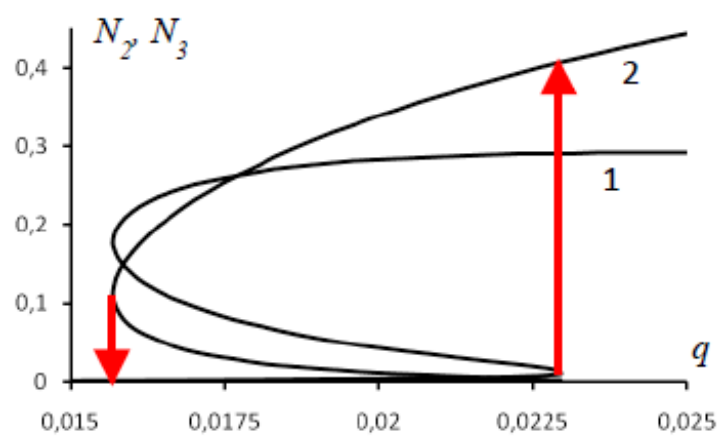

Fig. 6. Dependencies of occupancy of levels 2 (curve 1) and 2 (curve 2) with

$$
\text { the control parameter q; } \alpha^{1}=10 ; \alpha^{2}=100, \lambda_{2}=3, \lambda_{3}=50 \text {. }
$$

As the reduced number of system elements grows, the occupancy of all levels change abruptly (right arrows in Fig. 5. and Fig. 6.), and there is a drop in the occupancy of the upper level to almost zero. In contrast, the upper level occupancy increases abruptly to a comparatively high value (approximately till half of all the elements of the system). This can be interpreted as the discontinuous formation of a network from previously scattered elements. A similar pattern (Fig. 7. and Fig. 8.) occurs for the high values $\alpha^{1}$.

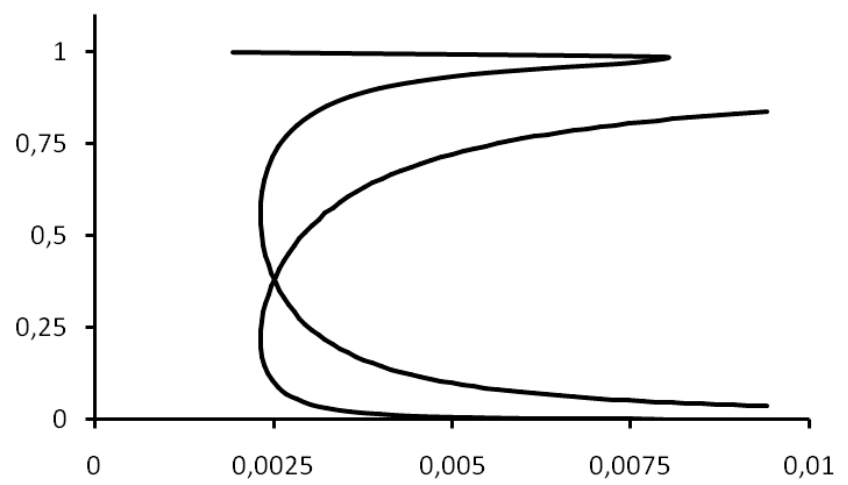

Fig. 7. Dependencies of occupancy of levels of 0 (curve 1) and 3 (curve 2) with the control parameter q; $\alpha^{1}=10 ; \alpha^{2}=1000, \lambda_{2}=3, \lambda_{3}=100$.

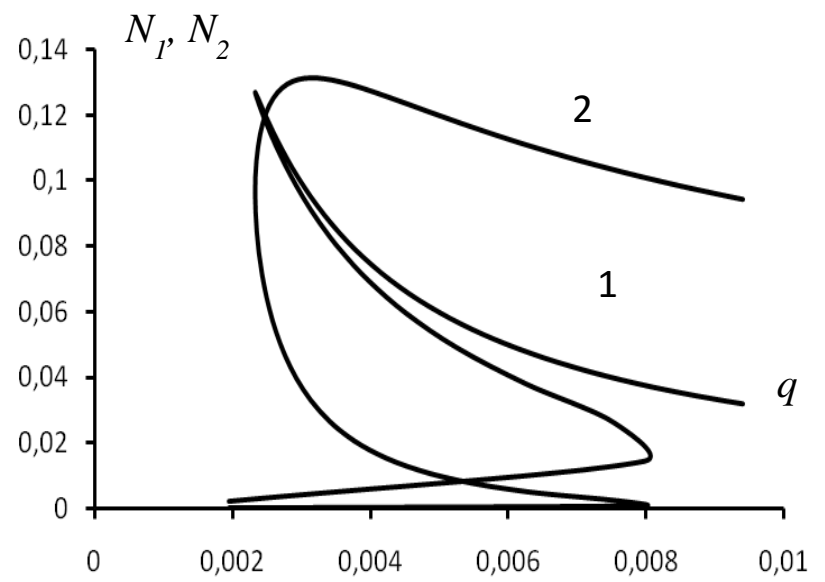

Fig. 8. Dependencies of occupancy of levels 1 (curve 1) and 2 (curve 2) with the control parameter q; $\alpha^{1}=10 ; \alpha^{2}=1000, \lambda_{2}=3, \lambda_{3}=100$.

\section{CONCLUSION}

Thus, in systems formed by elements of finite valence may also occur phase transitions, and for this type of systems is possible to obtain the explicit form of algebraic equations describing their condition.

It is essential that the phase transitions take place, including the systems with a very low valence of elements (four). The condition for the phase transition is occurred in a system which is a relatively high frequency of transition to the state corresponding to the formation of the maximum bonds.

\section{REFERENCES}

[1] R. Albert and A. L. Barabási, "Statistical mechanics of complex networks," Rev. Mod. Phys., vol. 74, no. 1, pp. 47-97, 2001.

[2] R. Pastor-Satorras and A. Vespignani, Evolution and Structure of Internet: A Statistical Physics Approach, Cambridge University Press, 2004.

[3] M. E. J. Newman, "The structure and function of networks," Comput. Phys. Commun., vol. 147, pp. 40-45, 2002.

[4] J. Marro and R. Dickman, Nonequilibrium Phase Transitions in Lattice Models, pp. 1417-1418, 1999.

[5] D. W. D. Wang et al. "Phase transition in complex networks," in Proc. Am. Control Conf. 2009, pp. 3310-3313.

[6] V. N. Zadorozhnyi and E. B. Yudin, "Growing network: Models following nonlinear preferential attachment rule," Phys. A Stat. Mech. its Appl. Elsevier B.V. vol. 428, pp. 111-132, 2015.

[7] G. Caldarelli et al. "Scale-Free networks from varying vertex intrinsic fitness," Physical Review Letters, vol. 89, Iss. 25, December 2002.

[8] G. L. Ciampaglia, E. Ferrara, and A. Flammini, "Collective behaviors and networks," EPJ Data Sci., 30 December, 2014.

[9] R. Pastor-Satorras and A. Vespignani, "Epidemic dynamics and endemic states in complex networks," Phys. Rev. E. Stat. Nonlin. Soft Matter Phys., vol. 63, 066117, 2001.

[10] I. Suleimenov, O. Güven, G. Mun, A. Beissegul, S. Panchenko, and R. Ivlev, "The formation of interpolymer complexes and hydrophilic associates of poly (acrylic acid) and non ionic copolymers based on 2 hydroxyethyl acrylate in aqueous solutions," Polymer International, vol. 62, no. 9, pp. 1310-1315, 2013.

[11] I. Suleimenov, D. Shaltykova, Z. Sedlakova, G. Mun, N. Semenyakin, D. Kaldybekov, and P. Obukhova, "Hydrophilic interpolymer associates as a satellite product of reactions of formation of interpolymer complexes," Applied Mechanics and Materials, vol. 467, pp. 58-63, 2014. 


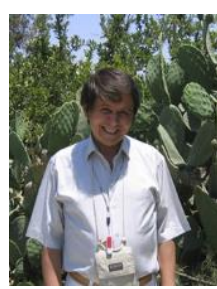

Ibragim E. Suleimenov is Head of the Laboratory of nanoelectronics of Almaty University of Power Engineering and telecommunications.

$\mathrm{He}$ is the Doctor of Chemical Sciences, Professor. Author of more than 400 scientific works and inventions. He is a recognized expert in the field of physical optics, plasma physics, who have made a huge contribution to the development of physics and chemistry of atmosphere.

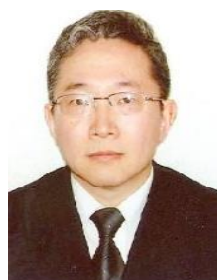

Grigoriy A. Mun is Head of the department of chemistry and technology of organic matters, natural compounds and polymers at the Al-Farabi Kazakh National University.

$\mathrm{He}$ is the Doctor of Chemical Sciences, Professor Author of more than 450 scientific, publications, including 2 scientific discoveries, 4 monographs, 2 manuals, 15 invention certificates, pre-patents, innovation patents. Winner of the state scientific grant for scientists and experts who have made outstanding contributions to the development of science and technology.

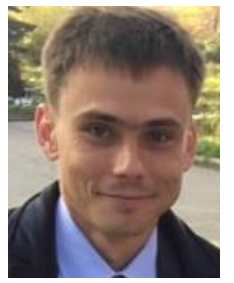

Sergey V. Panchenko was born in 1987 near Almaty city, Kazakhstan.

$\mathrm{He}$ is a bachelor of science in Radioengineering, electronics and telecommunications, Master of Science in Radioengineering, electronics and telecommunications and PhD student in department of Physics and Technology at al-Farabi Kazakh National University.

His main scientific interests are complex systems, computer simulation, information technologies. He has over 60 scientific publications including 3 monographs. Also he has professional experience as lead of IT department and senior lecturer in department of
Infocommunication Technologies of Almaty University of Power Engineering and telecommunications.

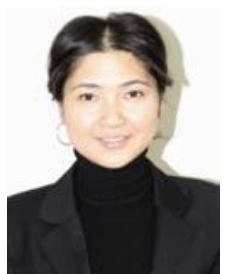

Zarina S. Tasbulatova has Master of science degree in Radioengineering, electronics and telecommunications from Almaty University of Power Engineering and Telecommunications, Post-master degree in Management and Contemporary Word (MBA) from Institute d'Etude Politique (Sciences Po). Now she is a $\mathrm{PhD}$ student in department of Infocommunication Technologies at Almaty Institute of Power Engineering and Telecommunications. Her major field of study is innovation and management of innovation.

Professional experience includes project management, business and marketing analysis and she is working as a junior lecturer in department of Infocommunication Technologies of Almaty University of Power Engineering and Telecommunications. She manages CRM department in distribution company and works as well as a junior scientist in National Academy of Science of Republic of Kazakhstan.

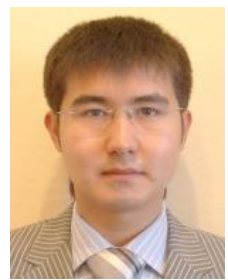

Anuar Nurtazin graduated from Moscow State University, M.S. in chemistry and economy.

$\mathrm{He}$ is the member of American Chemical Society and member of Professional Accountants Chamber.

$\mathrm{He}$ is the Winner of the state Prize for outstanding contributions to the development Eurasian Economic Union. Author of 18 scientific publications.

$\mathrm{He}$ is a recognized expert in the field of state audit and financial expertise. 